\title{
Diacronie
}

Studi di Storia Contemporanea

$N^{\circ} 7,3 \mid 2011$

«Spagna Anno Zero»: la guerra come soluzione

\section{La sorte del patrimonio artistico spagnolo durante la Guerra Civile (1936-1939)}

Ideologia, politica e trascendenza del problema

Rebeca Saavedra Arias

\section{(2) OpenEdition}

\section{Journals}

Edizione digitale

URL: http://journals.openedition.org/diacronie/3247

DOI: $10.4000 /$ diacronie.3247

ISSN: 2038-0925

Editore

Association culturelle Diacronie

Notizia bibliografica digitale

Rebeca Saavedra Arias, « La sorte del patrimonio artistico spagnolo durante la Guerra Civile

(1936-1939) », Diacronie [Online], № 7, 3 | 2011, documento 7, Messo online il 29 juillet 2011,

consultato il 01 mai 2019. URL : http://journals.openedition.org/diacronie/3247 ; DOI : 10.4000/ diacronie.3247

Creative Commons License 


\title{
Diacronie
}

\section{La sorte del patrimonio artistico spagnolo durante la Guerra Civile (1936-1939): ideologia, politica e trascendenza del problema* $^{*}$}

\author{
Rebeca SAAVEDRA ARIAS **
}

Durante la Guerra Civile il patrimonio artistico spagnolo fu in grave pericolo. I governi repubblicani, a differenza dei ribelli, coscienti di questa situazione ebbero una risposta pronta e ferma. La trascendenza internazionale della questione fece sì che fosse considerata un problema di Stato, intorno al quale si produsse un'autentica battaglia propagandistica. D'altra parte, l'intensa opera di comunicazione dei repubblicani per far conoscere tutto quanto era stato realizzato per assicurare la salvaguardia del patrimonio artistico ha permesso, oltre di migliorare la loro bistrattata immagine pubblica, di far conoscere le misure di conservazione adottate dalla Repubblica durante il conflitto. Questo processo sarebbe stato d'esempio per $i$ governi europei durante la II Guerra Mondiale.

l fallimento della sollevazione militare del 18 luglio del 1936 ebbe come prima
conseguenza l'inizio di una guerra tra le forze politiche e sociali che
appoggiavano i militari insorti e quelle che difendevano il governo stabilito

* Ho potuto migliorare quest'articolo grazie al contributo di Alessio Tarantino, Enrico Acciai e Giuseppe Fiorentini che mi hanno fornito un prezioso aiuto nella correzione e presentazione del testo in lingua italiana.

Questo articolo è stato realizzato grazie alla concessione di una borsa di studio e ricerca del Programa de FPU del Ministerio de Educación (Maggio 2008- maggio 2012). Le conclusioni che si offrono qui sono il risultato dell'analisi di documentazione proveniente dal Centro Documental de la Memoria Histórica (Salamanca), dall'Archivo General de la Administración (Alcalá de Henares), dall'Archivo General del Ministerio de Asuntos Exteriores (Madrid), dall' Archivo Diocesano de Santander (Santander) e dall'Archivo del Instituto del Patrimonio Cultural de España (Madrid). 
democraticamente nelle elezioni del febbraio del '36. Dopo gli scontri dei primi giorni, il paese restò diviso in due grandi retroguardie, nelle quali ci furono dei cambiamenti molto importanti nei modi di vita e nella socialità dei cittadini. Mentre nella zona della Spagna sotto il potere degli insorti si chiede un'ubbidienza cieca alle norme stabilite dai nuovi poteri militari e si torna ad uno stile di vita tradizionale, basato sui fondamenti morali della Chiesa Cattolica e sulle idee d'ordine e rispetto della proprietà privata; nella retroguardia repubblicana si sviluppa un processo di rivoluzione socioeconomica che sboccherà nell'atomizzazione del potere dello Stato repubblicano. La nascita di una miriade di poteri cittadini autonomi ha la sua origine nell'incapacità del governo del Fronte Popolare di contrastare la sollevazione servendosi soltanto delle forze coercitive dello Stato. L’incertezza sul risultato dell’insurrezione militare fece sì che lo spontaneo processo di mobilitazione popolare desse luogo alla nascita di comitati, giunte e consigli dal carattere locale oppure provinciale e dalla composizione eterogenea, i cui membri si arrogarono il potere d'amministrare, parallelamente al governo repubblicano, non de iure ma de facto i poteri esecutivo, giudiziario e militare. Queste iniziative strettamente vincolate con altre di carattere industriale e agricolo avevano come scopo quello di creare una società nuova, più egualitaria, che gettasse le sue basi sulle rovine della struttura sociale tradizionale che, fondata sulle differenze di classe, era riconosciuta dalla Chiesa e sostenuta da esercito e oligarchia. La trasformazione politica, economica e sociale che si visse nei primi mesi della guerra nella retroguardia repubblicana fu un'autentica sfida per il Governo Giral: obbligato a dare una risposta a tutte le situazioni proprie di una guerra e, allo stesso tempo, a rendere normale, per quanto fosse possibile, la vita della popolazione civile in tutto il territorio sotto il suo controllo ${ }^{1}$.

La distruzione del patrimonio storico-artistico si rivelò, sin dai primi giorni della guerra, un problema grave per le autorità repubblicane che dovettero intervenire rapidamente per evitare danni irreparabili. Come risultato dell'atomizzazione di poteri si produssero due fenomeni che misero in pericolo una parte dei beni culturali che si trovavano nella Spagna repubblicana: gli attacchi contro le proprietà della Chiesa e le requisizioni incontrollabili di collezioni d'arte e monumenti storico-artistici di proprietà privata. Le notizie della complicità di membri della Chiesa nell'organizzazione della sollevazione e la loro partecipazione ai primi scontri contro le forze leali alla Repubblica servirono per riaffermare un'idea condivisa dalle famiglie

\footnotetext{
1 Intorno alle origini della sollevazione e gli inizi della Guerra Civile è possibile consultare diversi testi, dallo studio classico di JACKSON, Gabriel, La República española y la guerra civil, Barcelona, Crítica, 1976, ai più recenti di PRESTON, Paul, La guerra civil española, Barcelona, Circulo de Lectores, 2006 o VIÑAS, Ángel, El desplome de la República, Barcelona, Crítica, 2009.
} 
politiche e sindacali della sinistra: la necessità di diminuire il potere della Chiesa. In virtù di questo si produsse la persecuzione dei membri del clero e la distruzione delle proprietà ecclesiastiche ${ }^{2}$. Se in alcuni casi, l'unico scopo degli assaltanti era bruciare, danneggiare o saccheggiare gli oggetti artistici e di culto che possedevano gli edifici ecclesiastici, in altri, era distruggere tutto, compreso lo stesso edificio. Sculture di legno, dipinti, altari, casule, stole, dalmatiche, ecc., erano distrutte con accanimento perché agli occhi del popolo rappresentavano simbolicamente e materialmente la dottrina delle Chiesa e i metodi usati quotidianamente durante secoli per imporla3. Gli edifici, se privati della loro decorazione, quando restavano in piedi, potevano essere secolarizzati. Così, durante la guerra, si potevano trovare chiese usate come magazzini, cinema o poste. Quando una Chiesa era attaccata e gli oggetti artistici sfuggivano alla distruzione, questi venivano confiscati per la riutilizzazione dei loro materiali oppure per essere venduti, visto e considerato che i beni artistici hanno un valore commerciale stabile, la loro compravendita poteva diventare una fonte d'entrata molto importante per i nuovi poteri. La realtà fu molto varia, ogni attacco era diverso, l'ira del popolo non colpiva allo stesso modo in tutta la geografia repubblicana. Lì dove il movimento anarchico era più forte, come in Catalogna e nei suoi dintorni, la maggior parte delle chiese soffrirono gravi danni. Ma anche in altre zone, come la Cantabria, dove la presenza dei movimenti della sinistra più radicale non aveva molto peso, si produssero incidenti di questo tipo in quasi tutti i paesi e villaggi della provincia, sebbene, in misura minore. Normalmente si è individuato negli anarchici e nelle "turbe incontrollabili”, i principali protagonisti delle aggressioni contro la Chiesa; di solito le testimonianze accusano gruppi di milizie di forestieri che quando arrivavano cominciavano il loro lavoro impartendo quello che si chiamava "la giustizia del popolo", ovvero la detenzione e, in molti casi, l'assassinio di chi era accusato di essere fascista (una categoria nella quale rientravano quasi sempre i membri della comunità ecclesiastica), la requisizione delle loro proprietà e la distruzione del loro patrimonio. Nonostante ciò, è giusto chiarire che sebbene la partecipazione degli anarchici in questi attacchi sia innegabile, visto che furono molti quelli che avrebbero ammesso di aver appoggiato e di aver presto parte a questo tipo d'attività, a mio giudizio si è esagerata la

2 Si vedano DE LA CUEVA MERINO, Julio, «Políticas laicistas y movilización anticlerical durante la segunda república y la guerra civil», In SUÁREZ CORTINA, Manuel (Ed.), Secularización y laicismo en la España contemporánea, Santander, Sociedad Menéndez Pelayo, 2001; LEDESMA, José Luís, «Qué violencia para qué retaguardia o la República en guerra de 1936», Ayer, no 76/ 2009 (4); CRUZ, Rafael, En el nombre del pueblo. República, rebelión y guerra en la España de 1936, Madrid, Siglo XXI, 2006.

3 Su questo argomento si veda DELGADO, Manuel, Luces iconoclastas. Anticlericalismo, blasfemia y martirio de imágenes, Barcelona, Ariel, 2002. 
loro responsabilità: da una parte, in base, al tradizionale appoggio dell'anarchismo all'utilizzazione della violenza e l'azione diretta come mezzo per raggiungere un obiettivo; dall'altra, ricorrendo a discorsi che hanno la loro origine nelle campagne di screditazione che contro l'anarchismo fecero prima gli insorti e poi, dopo del '37, anche i servizi di propaganda repubblicana. È un dettaglio indicativo in questo senso che in buona parte delle testimonianze riscontrabili dalle fonti appaiono le stesse formule per descrivere avvenimenti diversi. Non si può, inoltre, dimenticare come l'anticlericalismo non fosse esclusivo dell'ideologia anarchica, ma che, con sfumature diverse, fosse un elemento comune alle ideologie di tutte le forze di sinistra.

Se tralasciamo le tesi ampiamente diffuse dalla propaganda franchista che cercavano di presentare i sostenitori della Repubblica come barbari distruttori d'Arte, si vedrà come gli attacchi perpetrati contro i beni artistici della Chiesa non si possano definire come aggressioni contro la creazione artistica, ma bensì come un atto volto a cancellare tutte le tracce di quell'istituzione che si era storicamente occupata di definire le relazioni sociali e la morale pubblica e che ora era sotto attacco. Questo spiega, in parte, perché episodi del genere si estesero per tutto il territorio repubblicano. Si deve pure scartare la teoria che li presenta come "orde marxiste distruttrici” poiché né musei né altre istituzioni culturali pubbliche, furono attaccate dai civili o dai miliziani, neppure quando questi centri erano, nella Spagna degli anni Trenta, frequentati quasi esclusivamente dalle classi alte. Nonostante ciò, anche se le intenzioni non erano quelle di danneggiare il patrimonio artistico in quanto tale, alcuni degli effetti della rivoluzione sociale minacciarono direttamente la sua sopravvivenza. Parallelamente agli attacchi contro la Chiesa, i comitati operai e sindacali cominciarono a requisire le proprietà di quelli che si credeva simpatizzassero con gli insorti. Furono requisiti palazzi e appartamenti privati che conservavano opere d'arte di grande valore o che di per sé potevano essere considerati monumenti storico-artistici. La mancanza di un controllo rigoroso delle requisizioni, sommato al saccheggio delle chiese, mise nelle mani di queste organizzazioni una grande quantità di oggetti artistici, che, essendo fuori dal controllo delle leggi e della pubblica amministrazione, finirono per ingrossare l'elenco degli oggetti che si vendevano illegalmente nel ricco mercato nero d'opere d'arte del periodo inter-bellico. L'importante incremento dell'espatrio illecito d'arte di provenienza spagnola preoccupò entrambe le retroguardie, giacché le autorità erano coscienti della perdita irreparabile che questo comportava per l'eredità culturale 
spagnola4. Il problema si complicava perché, spesso, i contrabbandieri d'arte erano coinvolti in altre attività delittuose e ciò contribuiva a prolungare le indagini; inoltre, gli appelli alla collaborazione per frenare la compravendita illegale d'arte spagnola all'estero condotte dal governo della Repubblica e da quello di Burgos non ebbero grandi risultati, come dimostrato dal fatto che durante tutta la guerra entrambi i governi chiesero aiuto su questi temi ai governi stranieri.

Accanto alla distruzione del patrimonio artistico della Chiesa, alle requisizioni incontrollate, ai saccheggi, alla vendita illegale e all'espatrio delle opere d'arte, l'altro grande problema che mise in pericolo il patrimonio spagnolo durante il conflitto fu la diffusione dei bombardamenti a tappeto sulle città e sui paesi, che se non causavano la distruzione dei monumenti quanto meno li danneggiava. Prima della Guerra Civile spagnola gli eserciti rispettavano la regola militare che impediva di bombardare la popolazione civile indifesa; la guerra spagnola dimostrò invece che violare questa regola era uno strumento efficace per minare la capacità di resistenza dell'avversario e quindi si generalizzò l'uso di questa tattica, che avrebbe, in un secondo momento, causato grossi danni al patrimonio europeo durante la Seconda Guerra Mondiale.

Una volta individuati i quattro grandi problemi che mettevano in pericolo il patrimonio artistico spagnolo, si deve segnalare fino a che punto essi colpirono ciascuna retroguardia se si vogliono capire nella loro complessa eterogeneità le risposte che misero in pratica da un lato il Governo di Burgos e dall'altro il Governo repubblicano per proteggere l'integrità del Tesoro Artístico Nacional ${ }^{5}$. In questo senso le fonti documentali rivelano che mentre l'espatrio illecito d'arte ebbe effetto su entrambe le retroguardie, gli attacchi contro la Chiesa colpirono soprattutto la parte repubblicana. Nel territorio facente parte della retroguardia "nacional" già dall'agosto del '36 gli attacchi alle chiese furono fatti isolati, accaduti principalmente in occasione dei primi scontri di luglio, quando le forze insorte ancora non avevano il suo controllo definitivo. Una volta che il paese restò diviso in due zone ben distinte, nella retroguardia, sotto il comando degli insorti, la Chiesa e le sue proprietà furono rigorosamente rispettate come era scontato nei confronti di uno dei loro principali alleati sul piano ideologico e sociale. Non possiamo dimenticare che era stata proprio la Chiesa spagnola a fornire agli insorti, durante l'estate del '36, l'idea sulla quale si

4 SAAVEDRA ARIAS, Rebeca, «El mercado negro de obras de arte durante la Guerra Civil española (1936-1939). Una propuesta de análisis para su estudio», in COLORADO CASTELLARY, Arturo (Ed.), Patrimonio, Guerra Civil y Posguerra. Congreso internacional, Madrid, Servicio de Publicaciones Universidad Complutense de Madrid, 2010, pp. 189-199.

5 Tesoro Artistico Nacional è un'espressione usata nella Spagna degli anni Trenta per riferirsi all'insieme del patrimonio storico-artistico nazionale. 
sarebbe costruita la loro legittimazione del colpo di stato contro la Repubblica: cioè la necessità di liberare la Spagna tradizionale, cattolica, dal pericolo del dominio marxista attraverso una crociata di liberazione nazionale. D’altra parte, l'unica caratteristica ideologica che condividevano tutte le forze sociali, politiche e militari della fazione "nacional" era quella di essere cattolici e, per questa ragione, fu la Chiesa quella che servì in primo luogo per omogeneizzarle sotto il comando della cupola militare ribelle.

Come gli attacchi contro le chiese, anche le requisizioni incontrollate si producevano soltanto nella retroguardia repubblicana. Nella retroguardia sollevata le confische di beni si fecero sotto controllo diretto della nuova autorità. Inoltre, saranno proprio i monumenti situati nella zona repubblicana quelli che soffriranno di più per i danni causati dai bombardamenti. La capacità distruttiva dell'aviazione ribelle era superiore a quella repubblicana in virtù dalla migliore formazione dei suoi piloti e all'aiuto bellico ricevuto da Germania ed Italia ${ }^{6}$. D'altra parte, non si può omettere che le alte cariche sollevate, quando li consideravano come punti strategici per la difesa del nemico, non esitavano a far bombardare importanti centri artistici e culturali, come il Museo del Prado o il Palazzo di Liria, mettendo in grave pericolo le raccolte artistiche lì conservate. Tra l'altro è importante segnalare che la Spagna urbana, dove si trovavano i principali centri dell'alta cultura, era rimasta, grazie all'appoggio della classe operaia nei combattimenti dalle prime giornate di luglio, leale alla Repubblica e per questa ragione il suo governo si trovò a dover salvaguardare la maggior parte della ricchezza artistica del paese.

Una volta evidenziati i problemi e come i loro effetti fossero differenti a seconda della retroguardia considerata, conviene segnalare che le risposte avviate dai governi repubblicani e da quello di Burgos per assicurare la conservazione del patrimonio artistico ebbero tempi e modi diversi perché furono costruite in funzione dei loro differenti obiettivi prioritari che, a loro volta, erano il riflesso di due ideologie molto diverse sul modo di valutare l'importanza della Cultura per lo sviluppo della società spagnola. Mentre il Governo di Burgos aveva come principale, e quasi unico, scopo vincere la guerra prima di impegnarsi in qualunque altra iniziativa, per i governi repubblicani c'erano anche altre aspirazioni considerate di massima importanza. Si deve, a questo proposito, ricordare il mantenimento delle politiche culturali sviluppate

6 Cfr. BLANCO ESCOLÁ, Carlos, La academia general militar de Zaragoza (1928-1931), Barcelona, Labor Universitaria-Monografías, 1989; PRESTON, Paul, La República asediada. Hostilidad internacional y conflictos internos durante la guerra civil, Barcelona, Península, 2001; UXO PALASí, José, «La formación de oficiales para el ejército republicano durante la guerra civil (1936-1939)», in $V$ Congreso de Historia Militar: La enseñanza militar en España. 75 años de la Academia General Militar de Zaragoza, Zaragoza, Ministerio de Defensa, 2003; HEIBERG, Morten, Emperadores del Mediterráneo: Franco, Mussolini y la guerra civil española, Barcelona, Crítica, 2003. 
durante il primo biennio repubblicano per ridurre l'analfabetismo, la diffusione della cultura tra il popolo o la miglioria della tutela statale del patrimonio artistico nazionale. D'altra parte, è importante risaltare come tutti i progetti rivolti alla tutela del patrimonio si modificarono durante la guerra in base alle differenti necessità pratiche $\mathrm{e}$ materiali cui dovettero fare fronte tra il 1936 e il 1939 entrambi governi.

Appena sei giorni dopo il 18 luglio del '36 il governo della Repubblica decretò la creazione di un organismo che si occupasse della protezione della ricchezza artistica nazionale. L'idea era venuta dai membri dell'Alianza de Intelectuales Antifascista para la Defensa de la Cultura ${ }^{7}$ i quali, preoccupati per la sorte che avrebbero potuto correre le raccolte d'arte pubbliche e private in quella situazione, decisero di fare una proposta al governo ${ }^{8}$. La creazione della Junta aveva come fine l'intervento di questo organismo «con amplias facultades cuando objetos de arte o históricos y científicos se encuentren en los palacios ocupados, adoptando aquellas medidas que considere necesarias a su mejor conservación e instalación y trasladándolas provisionalmente, si así lo estimare, a los Museos, Archivos y Bibliotecas del Estado»9. Per decreto del primo d'agosto si ristrutturò la Junta originale che, da allora, si sarebbe chiamata Junta de Incautación y Protección del Tesoro Artístico ${ }^{10}$, avrebbe avuto più membri e avrebbe disposto d'una dotazione economica propria. La qualifica dei suoi membri, celebri restauratori e conservatori museali ma anche famosi artisti e intellettuali, e il loro impegno verso la causa per la quale lavoravano, la difesa dell'arte, fa del loro lavoro - nella misura permessa dalle circostanze della guerra e dalle disposizioni dei successivi governi repubblicani - la principale opera di salvaguardia del patrimonio artistico svolta

$7 \quad$ L'Alianza de Intelectuales Antifascistas para la Defensa de la Cultura nasce come sezione spagnola dell'Associazione Internazionale di Scrittori Antifascisti. Questa era stata creata nel ' 35 da un gruppo d'intellettuali europei che avevano partecipato nel '34 a Mosca al I Congresso di Scrittori Sovietici. Cfr. AZNAR, Manuel, «El Partido Comunista de España y la literatura (1931-1936)», in MAURICE, Jacques, MAGNIEN, Brigitte, GEVEVOIS, Bussy (dir.), Pueblo, movimiento obrero y cultura en la España contemporánea. Culturas populares, culturas obreras en España entre 1840 y 1936, Saint- Denis, Presses Universitaires de Vincennes, 1990.

8 Secondo la testimonianza di María Teresa León «la idea de esta protección nació entre los escritores de la Alianza de Intelectuales Antifascistas, [...] El Gobierno había confiado a la Alianza de Intelectuales un palacio abandonado por sus dueños, el del marqués de Heredia Spínola [...] Aquel palacio guardaba, en una de sus alas, la biblioteca más completa de Madrid en obras literarias hasta fines del siglo XIX. [...] En fin; los escritores, reunidos en esa biblioteca magnífica, abierta al sol y reintegrada a la vida, pensaron en la suerte posible de tantas maravillas como se recataban detrás de los palacios y celosías de conventos, y creo fue José Bergamín el que propuso se crease un organismo para protegerlas». Si veda in LEÓN, María Teresa, La Historia tiene la palabra (Noticias sobre el Salvamento del Tesoro Artístico), Madrid, Editorial Hispamerca, 1977, pp. 31-32.

9 Si veda ÁLVAREZ LOPERA, José, «La Junta del Tesoro Artístico de Madrid y la protección del patrimonio en la Guerra Civil», in ARGERICH, Isabel, ARA, Judith (Ed.), Arte protegido. Memoria de la Junta del Tesoro Artístico durante la Guerra Civil, Madrid, Instituto del Patrimonio Cultural de España y Museo Nacional del Prado, 2009, p. 29.

10 Ibidem. pp. 29-30. 
durante il conflitto. Tra i loro compiti c'erano da una parte la localizzazione e la supervisione del grado di conservazione delle collezioni requisite nei grandi palazzi della nobiltà e dell'alta borghesia, e dall'altra, la raccolta, l'inventario e l’immagazzinamento delle opere d'arte ma anche del patrimonio bibliografico e storico che fosse in pericolo di andare perso o essere distrutto nella zona d'interesse; spesso, se un'opera soffriva danni che potevano diventare irreversibili si facevano restauri d'urgenza. Per ridurre, inoltre, i danni prodotti dai bombardamenti si costruirono parapetti e protezioni per fontane, palazzi o facciate monumentali.

Nonostante ciò, al Ministerio de Instrucción Pública y Bellas Artes ${ }^{11}$ e nella stessa Junta erano coscienti che la loro attività sarebbe stata inutile se non fossero riusciti a coinvolgere la popolazione civile e, soprattutto, le forze operaie e sindacali nella conservazione del patrimonio. Quindi, con il fine d'ottenere la collaborazione cittadina promossero una campagna per sensibilizzare la popolazione ad una maggiore l'attenzione per l'Arte. Si deve pensare che nella Spagna degli anni Trenta il tasso d'analfabetismo oscillava tra il $30 \%$ ed il $50 \%{ }^{12}$; ciò costituiva un freno per la modernizzazione ed il normale sviluppo del paese. Benché le riforme educative messe in pratica dopo il '31 fossero riuscite a ridurre il numero di spagnoli che non sapevano né scrivere né leggere, non si erano ancora diffusi saperi più specifici, come la Fisica o la Storia dell'Arte. Quando scoppiò la guerra riemersero tutte le contraddizioni di una società che, uscendo dell'analfabetismo, si trovava in una fase di trasformazione; per questo, non ci si deve meravigliare del fatto che affiorarono comportamenti ereditati dal passato che, come il rogo delle chiese, si ripetevano ora adattati alla nuova realtà bellica. Nonostante la campagna stampa e radio realizzata per trasmettere la grande importanza del salvataggio del patrimonio storico-artistico per il futuro sviluppo culturale della nazione spagnola, la situazione non migliorava quanto ci si auspicava. I miliziani, ma anche i comuni e, dopo il settembre del '36, la Caja General de Reparaciones andarono avanti con le requisizioni di palazzi, appartamenti, macchine, fabbriche, e tutto quanto fosse appartenuto ai nemici della Repubblica e della rivoluzione; ciò, nella pratica, impedì il normale sviluppo dei lavori di cui era incaricata la Junta. Man mano che il governo repubblicano riusciva a recuperare i poteri e le competenze dello Stato, si emettevano decreti volti, da una parte, a ristrutturare la

\footnotetext{
11 Ministero della Pubblica Istruzione.

12 SAMANIEGO BONEU, Mercedes, La política educativa de la segunda república durante el bienio azañista, Madrid, CSIC Escuela de Historia Moderna, 1977; HOLGUIN, Sandie, República de ciudadanos. Cultura e identidad nacional en la España republicana, Barcelona, Crítica, 2003.
} 
Junta13, che dall'inizio del '37 ebbe una serie di Juntas Delegadas e Subjuntas locales ufficialmente riconosciute e destinate ad omogeneizzare le diverse iniziative che si stavano realizzando per mettere in salvo il patrimonio; e dall'altra, si cercava di chiarire quali fossero le funzioni delle Juntas e fin dove arrivassero le sue responsabilità. Nonostante i decreti e le buone intenzioni, l'incapacità dei governi repubblicani del periodo bellico, di separare in maniera inequivocabile le competenze sul patrimonio artistico del Ministerio de Instrucción Pública y Bellas Artes da quelle del Ministerio de Hacienda ${ }^{14}$ determinò litigi giurisdizionali continui. Le decisioni prese dai pubblici poteri in tale ambito furono sempre controverse, dal momento che, in molti casi, non furono capite né da tutti quelli che lavoravano per essi né dai loro avversari. Quando, alla fine dell'ottobre del '36, ebbe inizio l'assedio delle truppe di Franco a Madrid ed i bombardamenti si fecero quotidiani, il governo, convinto che la città non avrebbe potuto resistere per molti giorni all'avanzata dei ribelli, decise di abbandonare la capitale e di continuare il suo lavoro da Valencia; considerato che non poteva assicurare la protezione delle opere d'arte che rimasero immagazzinate a Madrid risolse la questione portando con sé a Valencia una selezione delle migliori opere d'arte che aveva in custodia. Il 10 novembre cominciò l'evacuazione del Tesoro Artístico Nacional nella quale, in diverse fasi, dalla fine del '36 al febbraio del ' 38 , si trasferirono in camion opere d'arte e reperti archeologici da Madrid a Valencia ${ }^{15}$, dove furono immagazzinate in immobili adattati a questo fine. Nell'ottobre del '37, dopo la caduta del Fronte Nord, il Governo decise di traslocare a Barcelona, però, in questa occasione, il patrimonio artistico non lo seguì. Appena quattro mesi dopo, tra la fine di febbraio e i primi giorni di marzo del ' 38 , le paure riaffiorarono quando, di fronte all'imminenza della frattura della retroguardia repubblicana in due parti, crebbe la preoccupazione per le opere che erano state lasciate a Valencia. I continui rovesci subiti dalle forze repubblicane

13 Intorno all'organizzazione e l'opera delle Juntas si possono consultare le testimonianze di RENAU, Josep, Arte en peligro (1936-1939), Valencia, Ayuntamiento de Valencia - Fernando Torres, 1980; LINO VAAMONDE, José, Salvamento y protección del Tesoro Artístico Español durante la guerra, 1936-1939, Caracas, Talleres de Cromotip, 1973; oppure le ricerche di ÁLVAREZ LOPERA, José, La política de bienes culturales del gobierno republicano durante la guerra civil español, Madrid, Ministerio de Cultura, 1982, Vol. 1 e ID., La política de bienes culturales del gobierno republicano durante la guerra civil española, Madrid, Ministerio de Cultura, 1982, Vol. 2 ; ARGERICH, Isabel, ARA Judith (Ed.), op. cit.

14 Ministero del Tesoro e delle Finanze.

15 Per uno studio più approfondito dell'evacuazione si veda CHACEL, Rosa, Timoteo Pérez Rubio y sus retratos del Jardín, Madrid, Cátedra, 1980, p. 102; LEÓN, María Teresa, op. cit., p. 42; ÁLVAREZ LOPERA, José, La política de bienes culturales del gobierno republicano durante la Guerra Civil española, Vol. II, Ministerio de Cultura, 1982; TUSELL, Javier, «El patrimonio artístico español en tiempos de crisis», in ARGERICH, Isabel, ARA Judith (Ed.), op. cit., p. 22; COLORADO CASTELLARY, Arturo, Éxodo y exilio del arte. La odisea del Museo del Prado durante la Guerra Civil, Madrid, Cátedra, 2008. 
avevano fatto crollare quasi tutte le speranze riposte nella capacità dell'esercito popolare di fare fronte alle truppe nemiche; per questo, davanti all'impossibilità di assicurare la conservazione delle opere durante la battaglia e del successivo esodo, si ordinò di evacuarle in Catalogna, anche se l'operazione rappresentava un pericolo e doveva essere fatta in maniera improvvisata.

In confronto con la complicata situazione che stava attraversando l'amministrazione repubblicana per assicurare la conservazione del patrimonio artistico nazionale, la realtà nella retroguardia ribelle si presentava più tranquilla. A differenza di quello che era successo nella parte repubblicana, i dirigenti ribelli non si preoccuparono di stabilire nessuna misura indirizzata a mettere in salvo le opere artistiche del loro territorio dai pericoli rappresentati dagli scontri durante i primi mesi della guerra. Dobbiamo aspettare il 23 dicembre del 1936 per trovare la prima disposizione riguardante tale questione ${ }^{16}$. La lentezza nel realizzare un progetto per proteggere l'arte era sicuramente da mettere in relazione con il fatto che le principali figure tra gli insorti erano militari e non civili. La formazione accademica di questi aveva una serie di caratteristiche che allontanavano la loro sensibilità da qualunque rappresentazione artistica o culturale che non esaltasse nessuno dei loro principi ideologici: la religione, la nazione e la tradizione. La considerazione, inoltre, delle nuove tendenze modernizzatrici e del libero pensiero caratteristico degli intellettuali come un pericolo per la perpetuazione dei loro principi, li faceva diffidare delle loro proposte. Siccome furono artisti e intellettuali coloro i quali in primo luogo mostrarono preoccupazione per il tema, era logico che gli insorti non avessero una risposta rapida. Inoltre, i militari sollevati avevano come principale scopo quello di eliminare il regime repubblicano e per questo, qualunque altra faccenda era subordinata. A questi argomenti ideologici dobbiamo aggiungere che nella loro zona non si produssero assalti contro i beni della Chiesa né requisizioni di proprietà private, ossia, i due grandi problemi cui dovettero far fronte i repubblicani nelle prime settimane della guerra. Ad ogni modo, mentre le chiese della loro retroguardia erano rispettate, gli insorti

16 Sul lavoro di protezione de Beni Culturali nella fazione "nacional" si veda ALTED, Alicia, Política del Nuevo Estado sobre el Patrimonio Cultural y la Educación durante la guerra civil española, Madrid, Ministerio de Cultura, 1984; MONREAL Y TEJADA, Luís, Arte y Guerra Civil, La Val de Onsera, Huesca, 1999; gli articoli di DÍAZ FRAILE, Teresa, «Medidas para la protección del tesoro artístico durante la Guerra Civil: las Juntas de Incautación y el Servicio de Recuperación Artística», pp. 539-551, RINCÓN GARCÍA, Wilfredo, «Teruel 1938. Destrucción del patrimonio y aportaciones documentales», pp. 511-524 e RODRÍGUEZ PEINADO, Laura, «Los depósitos de arte del Servicio de Recuperación Artística de Madrid», pp. 569- 581, tutti in CABAÑAS BRAVO, Miguel, LÓPEZ-YARTO ELIZALDE, Amelia e RINCÓN GARCÍA, Wilfredo (Coords.), Arte en tiempos de guerra, Madrid, Consejo Superior de Investigaciones Científicas, 2009. 
distruggevano edifici religiosi in zona repubblicana, quando li consideravano obiettivi militari.

Il 23 di dicembre del 1936 si creano le Juntas de Cultura Histórica y del Tesoro Artístico, pochi giorni dopo, il 14 gennaio del '37 si costituisce il Servicio Artístico de Vanguardia che sarebbe dovuto intervenire tra le popolazioni appena conquistate per verificare quale fosse la situazione del loro patrimonio artistico. L'obiettivo delle Juntas de Cultura Histórica y del Tesoro Artístico era di mantenere in condizioni accettabili i monumenti e le opere d'arte che si custodivano nella retroguardia "nacional"; a differenza, però, di quello che succedeva nella parte repubblicana non si stimolò il lavoro di ricerca sulle opere né si valorizzarono con il fine di ottenerne una fruizione pubblica durante la guerra. Malgrado queste disposizioni, i risultati ottenuti non furono buoni, i mezzi materiali e tecnici erano scarsi, inoltre, le persone che lavoravano per questi organismi lo facevano come volontari, senza ricevere alcuno stipendio. Attraverso lo studio della documentazione prodotta dai massimi responsabili di questi organismi, si è constatato che c'era un'importante mancanza d'organizzazione. Generalmente, si lamentavano della scarsa coordinazione esistente tra le diverse sezioni e i loro agenti. Finalmente, il 22 d'aprile del '38 si creava mediante decreto del Ministerio de Educación franchista il Servicio de Defensa del Patrimonio Artístico e si istituiva il Servizio de Recuperación Artística. Sarà in questo momento, quando si deciderà la militarizzazione dei membri dei Servicios de Vanguardia, che evidenzierà, un'altra volta, la supremazia dei valori militari su quelli artistici, culturali o civili.

L'importanza della conservazione del Tesoro Artistico Nacional fu tale che trascese l'orbita delle politiche culturali della Spagna in guerra e finì per convertirsi in un problema di Stato, soprattutto per i governi repubblicani. Tra i risultati della rivoluzione sociale vissuta nella retroguardia repubblicana, si trovavano, come è stato già detto, la distruzione di una parte notevole del patrimonio artistico ecclesiastico e la mancanza di controllo su un gran numero d'opere artistiche requisite in maniera poco ortodossa. I servizi di propaganda ribelle seppero utilizzare questi avvenimenti per denigrare l'immagine della Repubblica. La valanga di critiche su come l'amministrazione repubblicana aveva gestito, durante l'estate del '36, l'ondata d'attacchi contro la Chiesa e il saccheggio di proprietà private, attraversò il mondo danneggiando gravemente la sua stessa immagine. L'enorme ripercussione di tutto quanto avveniva in Spagna era conseguenza diretta dell'ambiente prebellico mondiale; nel 1936 tanto i governi quanto l'opinione pubblica erano coscienti dell'influenza che avrebbe avuto per la stabilità mondiale il risultato della guerra civile spagnola. Lo sviluppo internazionale del conflitto, grazie alla partecipazione delle brigate 
internazionali e dei combattenti tedeschi ed italiani, risultò decisivo per l'internazionalizzazione mediatica della guerra. Tutto ciò che accadeva in Spagna era una notizia, e tanto i ribelli quanto i repubblicani lo sapevano. Con l'intenzione di approfittare di questa visibilità entrambi i governi diedero inizio a una guerra attraverso i mezzi di comunicazione, volta a guadagnarsi le simpatie dell'opinione pubblica mondiale e fu intorno alla gestione del patrimonio che si produsse una delle più rilevanti battaglie propagandistiche. Gli insorti, che avevano assunto il ruolo di difensori della Chiesa Cattolica, approfittarono degli attacchi per dimostrare la cattiva gestione della conservazione del patrimonio storico-artistico da parte dai repubblicani; accusarono, inoltre, direttamente i governi repubblicani di incitare a tali distruzioni e di essere coinvolti nell'uscita illecita delle opere d'arte della Spagna. I dirigenti della Repubblica, al contrario, consapevoli dell'impossibilità di rifiutare le imputazioni che riguardavano gli attacchi contro i beni della Chiesa e le requisizioni incontrollate, optarono per una linea propagandistica basata sulla diffusione positiva delle linee generali e sugli scopi raggiunti dalla propria politica di conservazione del patrimonio. Tanto dai servizi di propaganda come dal Ministerio de Instrucción Pública y Bellas Artes si sapeva che i buoni risultati del lavoro delle Juntas sarebbero stati la migliore arma per dimostrare sia l'intima preoccupazione e il compromesso della Repubblica con la Cultura sia la correttezza delle decisioni prese e delle tecniche usate; per questo, non ebbero dubbi circa la diffusione di quanto era stato fatto. Tra il 1936 e il 1939 fu pubblicata una grande quantità di notizie in sostegno di questo argomento nella stampa mondiale; inoltre, con lo scopo d'arrivare a tutti i settori della popolazione, si usarono altri mezzi come la radio, i manifesti, il cinema o i volantini. Da parte repubblicana si arrivò a organizzare una campagna di diffusione culturale che portò un importante numero di artisti ed intellettuali ad offrire conferenze e interviste in America ed Europa indirizzate a influenzare gli intellettuali stranieri attraverso l'informazione sulla politica culturale della Repubblica. Questo progetto cercava di far sì che gli ambienti intellettuali esercitassero pressione sui loro governi in favore della causa della Spagna repubblicana. Sebbene tutta questa intensa attività riuscì a migliorare la deteriorata immagine della Repubblica, non raggiunse l'obiettivo di provocare un cambiamento negli atteggiamenti politici dei governi stranieri. I cattolici, inoltre, non smisero di manifestare contro un possibile aiuto alla Repubblica, che vedevano come un regime ateo e rivoluzionario che cercava di eliminare la Chiesa per stabilire un satellite dell'URSS nell'occidente europeo. Non tutto, però, fu invano. La risonanza che avevano conseguito i propagandisti repubblicani fece sì che il problema del salvataggio del patrimonio artistico spagnolo fosse presente nei mezzi museali mondiali. Questo fatto 
ebbe due conseguenze rilevanti: da un lato, contribuì alla nascita di un comitato internazionale creato da alcune delle massime autorità museali del mondo per aiutare a proteggere il patrimonio spagnolo durante la fase finale della guerra. Con questo fine firmarono col governo repubblicano un accordo in virtù del quale le principali opere e i reperti archeologici immagazzinati e tutelati dal governo centrale in Catalogna, e nella pratica anche alcune di quelli custoditi dalla Generalitat ${ }^{17}$, sarebbero stati trasferiti a Ginevra fino alla fine della guerra ${ }^{18}$. Dall'altro, alla fine della guerra la comunità museale mondiale attestò che le misure e le tecniche di conservazione e restauro sviluppate e utilizzate dai repubblicani erano risultate essenziali per l'ottima conservazione delle opere, nonostante i continui traslochi e il costante pericolo vissuto durante tre anni; per questo, funsero da esempio durante la II Guerra Mondiale. Le strategie militari e il differente uso dell'armamento realizzato durante la Guerra Civile spagnola rispetto a quanto era successo durante la I Guerra Mondiale facevano presagire che le misure utilizzate tra il 1914 e il 1918 per mettere in salvo il patrimonio sarebbero state inutili se fosse scoppiato un conflitto mondiale. Verso il 1938 le autorità della Svezia cominciarono a costruire magazzini sicuri per le loro opere d'arte anche nei propri musei. Allo stesso tempo in altri paesi, come l'Inghilterra o la Francia, si svilupparono strategie di evacuazione e si studiarono i migliori metodi di conservazione. L'influenza di quanto era avvenuto in Spagna la troviamo allo stesso modo nel fatto che tra le persone scelte in Francia per proteggere il patrimonio artistico nazionale si trovassero quattro dei membri del comitato internazionale che aveva organizzato l'evacuazione dell'arte spagnola a Ginevra, due tra quelli, J. Jaujard e P. Schommer, riconobbero che il caso spagnolo era servito come esempio per questo compito $^{19}$. Scoppiato il conflitto mondiale iniziò un movimento d'arte su vasta scala; da una parte si trovavano i governi che cercavano di preservare le raccolte pubbliche traslocandole, come era accaduto in Spagna, e nascondendole dal furto dei nazisti, dall'altra c'erano i proprietari privati ed i mercanti d'arte che facevano lo stesso per mettere in salvo le proprie collezioni oppure approfittavano per negoziare con l'occupante, avido collezionista d'arte. Sotto questo aspetto, tra gli anni Trenta e i

17 La Generalitat di Catalogna e il Governo Basco avevano in virtù dei loro Estatutos de Autonomía tutte le competenze sui Beni Culturali catalani e baschi; quindi, saranno questi organismi, e non il governo centrale, quelli che organizzarono la loro protezione durante la guerra.

18 COLORADO CASTELLARY, Arturo, Éxodo y exilio del arte. La odisea del Museo del Prado durante la Guerra Civil, Madrid, Cátedra, 2008.

19 Cfr. GRANGER Catherine, «L'évacuation des collections des musées espagnols et français», in COLORADO CASTELlARY, Arturo (Ed.), Patrimonio, Guerra Civil y Posguerra. Congreso internacional, Madrid, Servicio de Publicaciones Universidad Complutense de Madrid, 2010, pp. 339-356. 
Quaranta si produssero grandi cambiamenti non soltanto in tutto ciò che riguardava i possessori delle migliori collezioni d'opere d'arte, ma addirittura nelle regole di compravendita comunemente stabilite nel mercato d'arte mondiale.

In conclusione, si può dire che il processo di salvaguardia del patrimonio artistico spagnolo durante la Guerra Civile fu ampliamente propagandato all'estero e studiato dalla comunità museale, artistica ed intellettuale mondiale prima che cominciasse la II Guerra Mondiale, servendo, in questo modo, come modello per alcuni suoi aspetti. Non si deve dimenticare, comunque, che le misure usate e le decisioni prese dai governi repubblicani e dai vertici ribelli suscitarono grandi controversie, le quali, poiché non furono accettate come adeguate da tutti, finirono per dare luogo ad un'intensa battaglia propagandistica intorno al tema. Nonostante la polemica, gli studi storici realizzati attraverso le testimonianze e le fonti documentali preservate, dimostrano che furono le Juntas de Incautación y Protección del Tesoro Artístico create dal governo repubblicano nell'estate del '36 i migliori strumenti, per la quantità e la qualità del loro lavoro, sviluppati durante la Guerra Civile al fine di proteggere i Beni Storico-Artistici e Bibliografici della Spagna. 


\section{** L'autore}

Rebeca Saavedra Arias si è laureata in Storia preso l'Università di Cantabria (Santander, Spagna), dove attualmente è ricercatrice specializzata in Storia Contemporanea. Ha conseguito il titolo di Master Europeo in Gestione e Conservazione dei Beni Culturali e Master Interuniversitario in Storia Contemporanea. Dal 2008 è ricercatrice borsista del Ministerio de Educación con lo scopo di sviluppare il suo progetto di tesi di dottorato El Patrimonio Artístico durante la Guerra Civil (1936-1939): política e ideología en las «dos Españas». Ha svolto stage nell'Università di Salamanca, preso i Musei Capitolini, ecc. Negli ultimi mesi ha collaborato nell'organizzazione del $X$ Congreso de la Asociación de Historia Contemporánea. Tra le sue publicazioni più recenti si trova «El Patrimonio Artístico español en 1939: ¿Cambio de rumbo en la política cultural republicana?», Tzintzun Revista de Estudios Históricos del Instituto de Investigaciones Históricas de la Universidad Michoacana de San Nicolás de Hidalgo (Morelia, Michoacán, México), número 50, julio-diciembre 2009. I suoi interessi spaziano dalla storia delle politiche culturali contemporanee, in particolare nel contesto spagnolo degli anni Trenta, ai temi degli usi propagandistici ed economici della Cultura.

URL: < http://studistorici.com/progett/autori/\#Saavedra >

\section{Per citare questo articolo:}

SAAVEDRA ARIAS, Rebeca, «La sorte del patrimonio artistico spagnolo durante la Guerra Civile (1936-1939): ideologia, politica e trascendenza del problema», Diacronie. Studi di Storia Contemporanea: Spagna Anno Zero: la guerra come soluzione, 29/07/2011,

URL:< http://www.studistorici.com/2011/07/29/saavedra_numero_7/ >

Diacronie Studi di Storia Contemporanea $\beta$ www.diacronie.it

Risorsa digitale indipendente a carattere storiografico. Uscita trimestrale. redazione.diacronie@hotmail.it

Comitato di redazione: Marco Abram - Giampaolo Amodei - Jacopo Bassi - Luca Bufarale - Alessandro Cattunar - Alice De Rensis Barbara Galimberti - Deborah Paci - Fausto Pietrancosta - Martina Sanna - Matteo Tomasoni - Luca Zuccolo

Diritti: gli articoli di Diacronie. Studi di Storia Contemporanea sono pubblicati sotto licenza Creative Commons 2.5. Possono essere riprodotti a patto di non modificarne i contenuti e di non usarli per fini commerciali. La citazione di estratti è comunque sempre autorizzata, nei limiti previsti dalla legge. 